\title{
Theta Oscillations in Human Cortex During a \\ Working-Memory Task: Evidence for Local \\ Generators
}

S. Raghavachari, J. E. Lisman, M. Tully, J. R. Madsen, E. B. Bromfield and M.

J. Kahana

$J N$ 95:1630-1638, 2006. First published Oct 5, 2005; doi:10.1152/jn.00409.2005

You might find this additional information useful...

This article cites 48 articles, 16 of which you can access free at:

http://jn.physiology.org/cgi/content/full/95/3/1630\#BIBL

Updated information and services including high-resolution figures, can be found at:

http://jn.physiology.org/cgi/content/full/95/3/1630

Additional material and information about Journal of Neurophysiology can be found at: http://www.the-aps.org/publications/jn

This information is current as of April 17, 2006. 


\title{
Theta Oscillations in Human Cortex During a Working-Memory Task: Evidence for Local Generators
}

\author{
S. Raghavachari, ${ }^{1}$ J. E. Lisman, ${ }^{1}$ M. Tully, ${ }^{2,4}$ J. R. Madsen, $^{2}$ E. B. Bromfield, ${ }^{3}$ and M. J. Kahana ${ }^{4}$ \\ ${ }^{1}$ Volen Center for Complex Systems, Brandeis University, Waltham; ${ }^{2}$ Department of Neurosurgery, Children's Hospital and \\ ${ }^{3}$ Department of Neurology, Brigham and Women's Hospital, Boston, Massachusetts; and ${ }^{4}$ Department of Psychology, \\ University of Pennsylvania, Philadelphia, Pennsylvania
}

Submitted 20 April 2005; accepted in final form 28 September 2005

\begin{abstract}
Raghavachari, S., J. E. Lisman, M. Tully, J. R. Madsen, E. B. Bromfield, and M. J. Kahana. Theta oscillations in human cortex during a working-memory task: evidence for local generators. $J$ Neurophysiol 95: 1630-1638, 2006. First published October 5, 2005; doi:10.1152/jn.00409.2005. Cortical theta appears important in sensory processing and memory. Intracanial electrode recordings provide a high spatial resolution method for studying such oscillations during cognitive tasks. Recent work revealed sites at which oscillations in the theta range $(4-12 \mathrm{~Hz})$ could be gated by a working-memory task: theta power was increased at task onset and continued until task offset. Using a large data set that has now been collected (10 participants/619 recording sites), we have sufficient sampling to determine how these gated sites are distributed in the cortex and how they are synchronized. A substantial fraction of sites in occipital/parietal (45/157) and temporal $(23 / 280)$ cortices were gated by the task. Surprisingly, this aspect of working-memory function was virtually absent in frontal cortex (2/182). Coherence measures were used to analyze the synchronization of oscillations. We suspected that because of their coordinate regulation by the working-memory task, gated sites would have synchronized theta oscillations. We found that, whereas nearby gated sites $(<20 \mathrm{~mm})$ were often but not always coherent, distant gated sites were almost never coherent. Our results imply that there are local mechanisms for the generation of cortical theta.
\end{abstract}

\section{N T R O D U C T I O N}

It is now well accepted that the brain displays oscillations in field potentials and that these oscillations reflect the synchronized rhythmic activity of large groups of neurons. Oscillations in the 4- to $12-\mathrm{Hz}$ theta-frequency range have been most extensively studied in the rat hippocampus (Buzsaki 2002), where they play a role in the phase-dependent coding of information (Jensen and Lisman 1998; O'Keefe and Recce 1993) and phase-dependent plasticity (Hölscher et al. 1997; Huerta and Lisman 1995; Pavlides et al. 1988). Procedures that interfere with hippocampal theta produce strong deficits in memory processes (Winson 1978). More recent work has made it clear that theta oscillations in rat are not confined to the hippocampus, but can also be recorded in many sensory regions of the rat cortex (Leung and Borst 1987; Silva et al. 1991).

Multielectrode intracranial EEG (iEEG) recordings have provided unequivocal evidence that theta oscillations also occur in the human cortex (Caplan et al. 2001; Howard et al. 2003; Kahana et al. 1999; Kahana 2006; Rizzuto et al. 2003; Sederberg et al. 2003), arising from the high signal-to-noise

Address for reprint requests and other correspondence: M. J. Kahana, Department of Psychology, University of Pennsylvania, Philadelphia, PA 19104 (E-mail: kahana@psych.upenn.edu). ratio of this method. Of particular interest is the finding (Raghavachari et al. 2001) that human theta can be engaged by the Sternberg (1966) working-memory task. At many cortical locations, theta oscillations are gated: theta power rises sharply when working memory becomes required, is maintained throughout the memory task, and decreases when working memory is no longer required. Furthermore, in experiments where successful retrieval depends on the long-term retention of items, theta power during encoding predicts subsequent recall (Sederberg et al. 2003). These results, which suggest that there is an oscillatory basis to working memory, make it important to determine the properties and locations of the gated sites engaged by working memory demands. Because of the limited number of patients studied and the small number of electrode grids, our previous study (Raghavachari et al. 2001) was unable to characterize the topography of theta gating. One goal of the current work was to use the larger participant sample now available to provide a description of the location of gated sites.

A second goal of the current work was to determine how brain regions that are jointly engaged by a task interact with each other. Such interactions may provide crucial insights into the ways in which oscillatory processes are generated within the human brain and how such oscillations serve to organize cognitive processes. Studies in the rat hippocampus showed that theta oscillations are synchronized throughout this brain structure (Bullock et al. 1990). An important unexplored question is whether the gated sites in human cortex that are commonly engaged while the participants performed the Sternberg task are similarly synchronized. Given their coordinated regulation by the task, the various gated sites could be part of a distributed network with strong synchronization between regions. Previous work on human EEG has shown task-dependent increases in theta synchronization (Sarnthein et al. 1998), suggesting that intersite coherence may be important. However, as a result of the low signal-to-noise ratio in scalp EEG recordings, it has not been possible to determine whether the absolute level of synchronization between brain regions is high or low. There are reasons to suspect that gated sites may not be synchronized. Cellular and network analysis of cortical slices (Bao and Wu 2003; Blatow et al. 2003; Flint and Connors 1996; Silva et al. 1991) suggest that small cortical regions have the mechanisms to generate theta locally, making it possible that even sites commonly engaged in working memory oscil-

\footnotetext{
The costs of publication of this article were defrayed in part by the payment of page charges. The article must therefore be hereby marked "advertisement" in accordance with 18 U.S.C. Section 1734 solely to indicate this fact.
} 

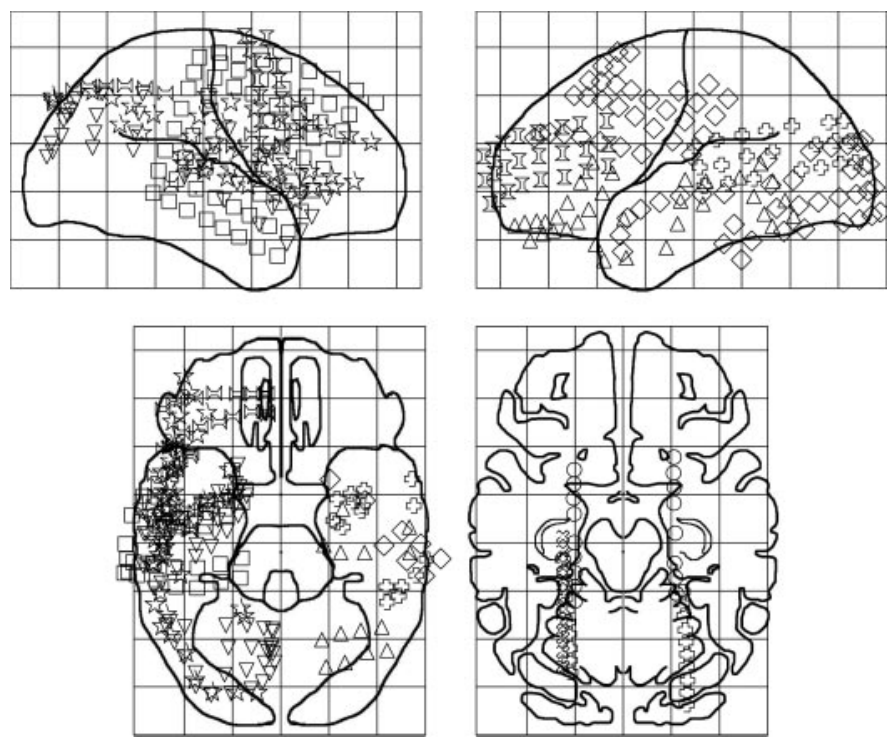

FIG. 1. Electrode locations. Topographic maps display electrode locations on 4 views of a standard brain. Top left: right lateral view. Top right: left lateral view. Bottom left: inferior view. Bottom right: axial slice through the hippocampus depicting the locations of all depth electrodes. Only electrodes included in subsequent analyses are shown (different shapes denote different participants)

late independently. We used iEEG signals, which have high signal-to-noise ratio, to determine the absolute level of theta synchronization among gated cortical sites. The results clearly show that distant gated sites are generally not synchronized.

\section{METHODS}

\section{Participants}

We tested ten participants who had implanted cortical surface (subdural) and/or bilateral depth electrodes. The clinical team determined the placement of these electrodes so as to best localize epileptogenic regions. The location of the electrodes was determined using coregistered postoperative computed tomograms (CTs) and preoperative magnetic resonance images (MRIs) by an indirect stereotactic technique. Across all ten participants, we analyzed iEEG data from 619 electrodes. Figure 1 illustrates the positions of the electrodes. All participants had normal range personality and intelligence and were able to perform the Sternberg task within normal limits (see Table 1). Our research protocol was approved by the institutional review boards at Children's Hospital, Boston and Brigham and Women's Hospital, Boston and informed consent was obtained from the participants and their guardians.

\section{Procedure}

The Sternberg task is a classic test of multiitem short-term memory (Sternberg 1966). The participant pressed a key to initiate each trial. An orienting stimulus (an asterisk) was then displayed in the center of a computer screen and remained visible for $1 \mathrm{~s}$. After a variable delay of $200 \pm 75 \mathrm{~ms}$ (uniformly distributed), four consonants were sequentially displayed. The temporal jitter was introduced to ensure that each stimulus arrived at a random phase with respect to ongoing oscillations, thus ensuring that the prestimulus phase was uniformly distributed. Each consonant was displayed for $700 \mathrm{~ms}$ followed by a variable delay of $275 \pm 75 \mathrm{~ms}$. Consonants were randomly selected, subject to the constraint that a particular consonant not repeat within three successive lists. The last (fourth) consonant was followed by a retention interval of $500 \pm 75 \mathrm{~ms}$, in turn followed by the presentation of the memory probe. The participant was instructed to indicate as quickly and accurately as possible whether the probe item either was in the preceding list (a target) or was not in the preceding list (a lure) by pressing the right control key to target items and the left control key to lures. Targets and lures occurred with equal probability, and target items were drawn equally from each of the list positions. Participants in the hospital setting performed the task with reasonable accuracy (see Table 1$)$ and with a mean reaction time $(<1 s)$ that was not much longer than that for normal participants under standard laboratory conditions (i.e., 550-750 ms). Because there were few error trials during this task $(<5 \%)$, we restricted our analysis to correct trials. Participants 7 and 8 did a version of the task where the stimulus was presented for $1 \mathrm{~s}$ and interstimulus delay was fixed at $400 \mathrm{~ms}$. The retention interval was fixed at $2.2 \mathrm{~s}$. Subjects exhibited a modest serial position effect, with slightly faster responses and lower error rates when probed with the most recent item (Rizzuto et al. 2003).

\section{iEEG recordings}

The iEEG signal was recorded from platinum electrodes $(3 \mathrm{~mm}$ diameter) with an interelectrode spacing of $10 \mathrm{~mm}$ (for subdural electrodes) or with a spacing of $8 \mathrm{~mm}$ (for depth electrodes). The signals were amplified, sampled at $256 \mathrm{~Hz}$ (Children's Hospital, Bio-Logic apparatus: Participants 1-8) or $200 \mathrm{~Hz}$ (Brigham and Women's Hospital, Nicolet Biomedical apparatus: Participants 9 and 10), and band-pass filtered (Bio-Logic, 0:3-70 Hz; Nicolet Biomedical, 0:5-60 Hz). For all participants, the locations of the electrodes were determined using coregistered postoperative CTs and preoperative MRIs by an indirect stereotactic technique (Caplan et al. 2003). The raw iEEG signal was referred to a skull-fixed electrode and no additional referencing was done. Electrodes showing artifacts were excluded using criteria described in Raghavachari et al. (2001).

TABLE 1. Participant demographics and performance

\begin{tabular}{|c|c|c|c|c|c|c|c|c|}
\hline Participant & Age & Gender & $\begin{array}{c}\text { Electrode } \\
\text { Placement }\end{array}$ & Resection & $\begin{array}{l}\text { Number of } \\
\text { Electrodes }\end{array}$ & $\begin{array}{l}\text { Number } \\
\text { Excluded }\end{array}$ & $\begin{array}{c}\text { Accuracy } \\
\%\end{array}$ & $\begin{array}{c}\text { Mean RT, } \\
\text { ms }\end{array}$ \\
\hline 1 & 17 & M & Subdural & $\mathrm{L}$ anterior temporal & 80 & 20 & 98 & 1168 \\
\hline 2 & 12 & $\mathrm{~F}$ & Subdural & $\mathrm{R}$ motor-sensory transection & 104 & 12 & 94 & 970 \\
\hline 3 & 14 & M & Depth & No resection & 19 & 2 & 99 & 1240 \\
\hline 4 & 21 & M & Depth/subdural & $\mathrm{R}$ temporal & 76 & 17 & 85 & 1232 \\
\hline 5 & 18 & M & Subdural & Anterior/temporal & 64 & 16 & 96 & 655 \\
\hline 8 & 15 & M & Subdural & $\mathrm{R}$ anterior temporal & 128 & 31 & 92 & 1158 \\
\hline 6 & 18 & M & Depth & R temporal & 20 & 10 & 90 & 800 \\
\hline 7 & 22 & $\mathrm{~F}$ & Subdural & L occipital & 96 & 18 & 95 & 620 \\
\hline 9 & 41 & M & Subdural & $\mathrm{R}$ temporal & 64 & 12 & 97 & 954 \\
\hline 10 & 38 & M & Subdural & R temporal & 94 & 4 & 98 & 1483 \\
\hline
\end{tabular}




\section{Spectral analysis}

Time-varying spectra (spectrograms) were computed using the squared modulus of the complex demodulates of the iEEG data using filters constructed from the Slepian windows of 1-s duration and 4-Hz bandwidth (see Raghavachari et al. 2001 for details). Estimates from different Slepian windows were averaged together to obtain the spectrogram for each trial. The spectrograms for each trial were aligned with the onset of the first list item and averaged together.

\section{Tests for gating}

Using a previously described test (Raghavachari et al. 2001), we found that the iEEG signals showed significant nonstationarity in the theta-frequency range, and that this nonstationarity corresponded to a continuous elevation of theta power during a trial of the Sternberg task with sharp onset and offset at the start and end of the trial, respectively (which we termed theta gating). We thus devised a test to detect sites that displayed gated theta during the Sternberg task (see Raghavachari et al. 2001 for details). Briefly, gating of theta was tested by comparing the spectral power in the $4-$ to $12-\mathrm{Hz}$ range in the average spectrogram in every overlapping 250 -ms epoch during the trial to the power in the intertrial period (1 s before the orienting stimulus). Because the distribution of power in the spectrogram is non-Gaussian, the values were log-transformed to reduce the effects of the tail of the distribution and then a nonparametric method (Mann-Whitney $U$ test, $P<0.01$ ) was used to compare the average power in each $250-\mathrm{ms}$ epoch during the trial with the power in the intertrial period. Because the analysis windows were $1 \mathrm{~s}$ long, adjacent 250-ms bins were not independent. Multiple comparisons were corrected for by a Bonferroni correction for the number of independent time bins.

\section{Coherence analysis}

The coherence between signals is a Fourier transform of the cross-correlation of two time-domain signals. An unbiased and consistent estimate of the coherence can be obtained by first windowing the data with $K$ Slepian windows and then calculating

$$
\gamma(f)=\sum_{k=1}^{K} \frac{X_{k}(f) Y_{k}^{*}(f)}{\left|X_{k}(f)\right|^{2}\left|Y_{k}(f)\right|^{2}}
$$

where $\gamma(f)$ is the coherence and $X_{k}(f)$ and $Y_{k}(f)$ are the Fourier transforms of the data from two channels windowed by the $K$ Slepian windows. Using these windows results in $K$ independent estimates of coherence. We used a sliding window (50\% overlap) with each segment of data windowed by three 1-s windows, each of which has a 4-Hz bandwidth (see Spectral analysis above). This choice of window length resulted in an estimate of coherence averaged over 24 degrees of freedom (corrected for the overlap) for each trial. Because of the large number of independent samples, our estimate of the coherence was consistent in a statistical sense (Zaveri et al. 1999). For Participants 7 and 8, the window length was increased to $1.3 \mathrm{~s}$ to maintain the same number of degrees of freedom for estimating coherence as that in other participants. The value of coherence lies between 0 and 1 , and its estimate from a single window is highly skewed and non-Gaussian. However, first using a variance-stabilizing transformation $\left(\tanh ^{-1}\right)$ and averaging the transformed values over different windows renders its distribution approximately normal (Thomson and Chave 1991). Thus for approximately Gaussian data, the SD of the coherence in the transformed units is about 1, allowing us to obtain tight confidence intervals. The median value of magnitude-squared coherence for uncorrelated Gaussian data sets calculated using 24 degrees of freedom is about 0.05 .

\section{Significance tests for coherence}

The distribution of coherence estimates for uncorrelated, Gaussian data has been shown to be

$$
\mathrm{p}\left(\gamma^{2}\right)=\frac{\left(1-\gamma^{2}\right)^{m-2}}{m-1}
$$

where $m$ is the number of independent samples used in the estimate (Thomson and Chave 1991). This forms a useful benchmark to compare values of coherence calculated using Eq. 1. However, iEEG data are best described as oscillatory activity in a red noise background (Mitra and Pesaran 1999). Thus we developed a nonparametric test for significance using a bootstrap technique. We permuted each data set randomly in time, but applied the same permutation to both time series. This procedure whitens the data set, but maintains any spatial relationships that are independent of frequency. We then estimated the coherence for this randomized data set. We repeated this procedure 1,000 times to obtain an empirical null distribution. The trial-averaged true coherence was judged to be significant if it exceeds the 99.9th percentile of the null distribution $(P=0.001)$.

\section{RES U L T S}

The iEEG signal was recorded from 619 electrodes across ten participants as they performed a Sternberg working-memory task (see Fig. $2 A$, METHODS) ${ }^{1}$. Electrodes were either arrayed on grids, which were implanted subdurally on the cortical surface, or along depth probes that penetrated medial temporal cortex from a posterior approach (Fig. 1). Electrode placement was entirely determined by clinical considerations and generally involved multiple grids and/or depth electrodes implanted in various brain regions of a given patient.

Of the 619 electrodes, 72 met our criteria for theta gating (see METHODS). At these sites, oscillatory power in the thetafrequency range sharply rose at the beginning of the trial, remained elevated throughout the trial, and returned to baseline after the participant's response. In Fig. $2 B$, we show an example of theta gating at a site in the parietal cortex from Participant 1 . The peak frequency of theta oscillations at this site was $8 \mathrm{~Hz}$. We found no tendency for theta frequency to gradually change during the gated period (Fig. 2B). We also observed that superimposed on the overall gating effect was a tendency for the theta power to transiently increase as each list item was presented. The peak frequency of theta oscillations across all gated sites from all subjects was $8 \pm 1 \mathrm{~Hz}$.

As previously found (Raghavachari et al. 2001) with a more limited sample, gated sites are widespread across brain regions (Fig. 2C). We found 33 gated sites (out of 115) in the occipital cortex, 14 (out of 42) in the parietal lobe, 12 (out of 200) in the temporal lobe, 11 (out of 80) in the hippocampal and parahippocampal regions, and two (out of 182) in the frontal cortex. Of particular note is the higher probability of gated sites in occipital cortex as well as parietal cortex compared with other cortical regions $\left(\chi_{4}^{2}=76, P<0.001\right)$. The occipital sites included those near the midline that would include early visual areas. Also of note is the very small percentage of gated sites in prefrontal cortex, a region implicated in working memory.

\footnotetext{
${ }^{1}$ The Supplementary Material for this article (three figures) is available online at http://jn.physiology.org/cgi/content/full/00409.2005/DC1.
} 
A

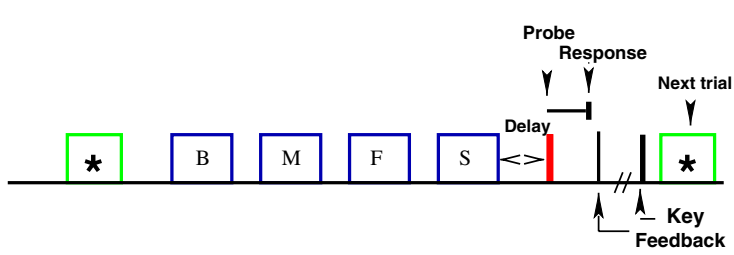

B

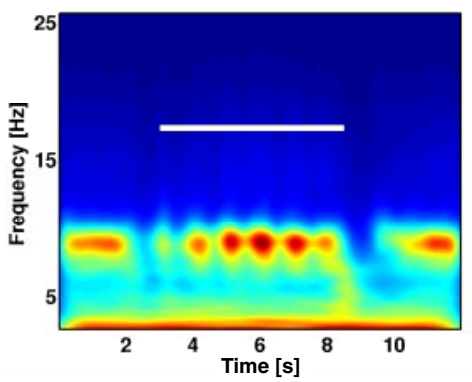

C

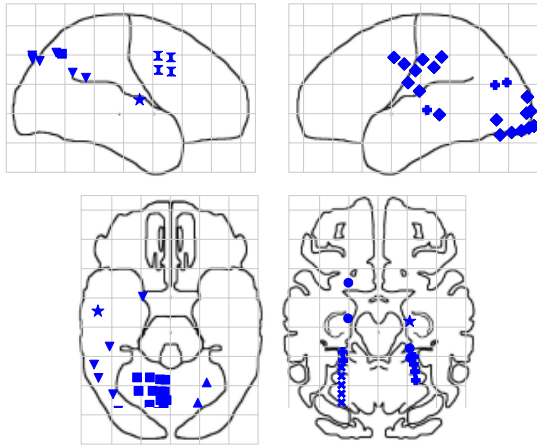

FIG. 2. Sternberg item recognition task gates human theta oscillations. A: illustration of the experimental design. Orienting stimulus (asterisk) appears first, followed by the 4 list items (consonants) and a delay. After the delay a probe is presented and the participant makes a response indicating whether the probe item was in the preceding list. $B$ : average spectrogram for all electrodes that met our gating criteria. White bar shows the mean extent of a trial. $C$ : symbols indicate the locations of sites exhibiting gated theta activity (different shapes denote different participants).

\section{Synchronization between sites}

A central question of the present study was whether the coordinated phenomenon of theta gating reflects the coherent action of a large oscillating network that supports working memory. Such an oscillatory network would be seen as synchronous oscillations between gated sites within a given participant. We first considered the synchrony of oscillatory activity between different electrode sites, irrespective of whether they exhibited gating. Computing the spectral coherence, a measure of shared variance between two signals, we examined coherence among different recording sites during the interval from onset of the first list item until the response. We found that the spectral coherence decreased as the Talairach distance between recording sites increased (Fig. 3A). The coherence had a peak in the theta-frequency $(4-12 \mathrm{~Hz})$ range. We similarly found increased theta coherence in all participants. To estimate the distances over which the iEEG coherence was significant, we developed a nonparametric estimate of spectral coherence using a bootstrap technique (see METHODS). We found that within the theta band, spectral coherence decayed to chance values at distances $>40 \mathrm{~mm}$ (Fig. $3 B$ ). More specifically, sites separated by distances $>60 \mathrm{~mm}$ have coherence values clus- tered around the median coherence values for two artificial, Gaussian-distributed time series. Across the entire data set, theta coherence decayed as a shallow function (power law) of interelectrode separation, as distance ${ }^{-0.31 \pm 0.02}$ (Fig. 3C). However, because Talairach distance is not a measure of the actual cortical distance between recording sites because of the highly convoluted nature of the cortex, the actual decay might be even
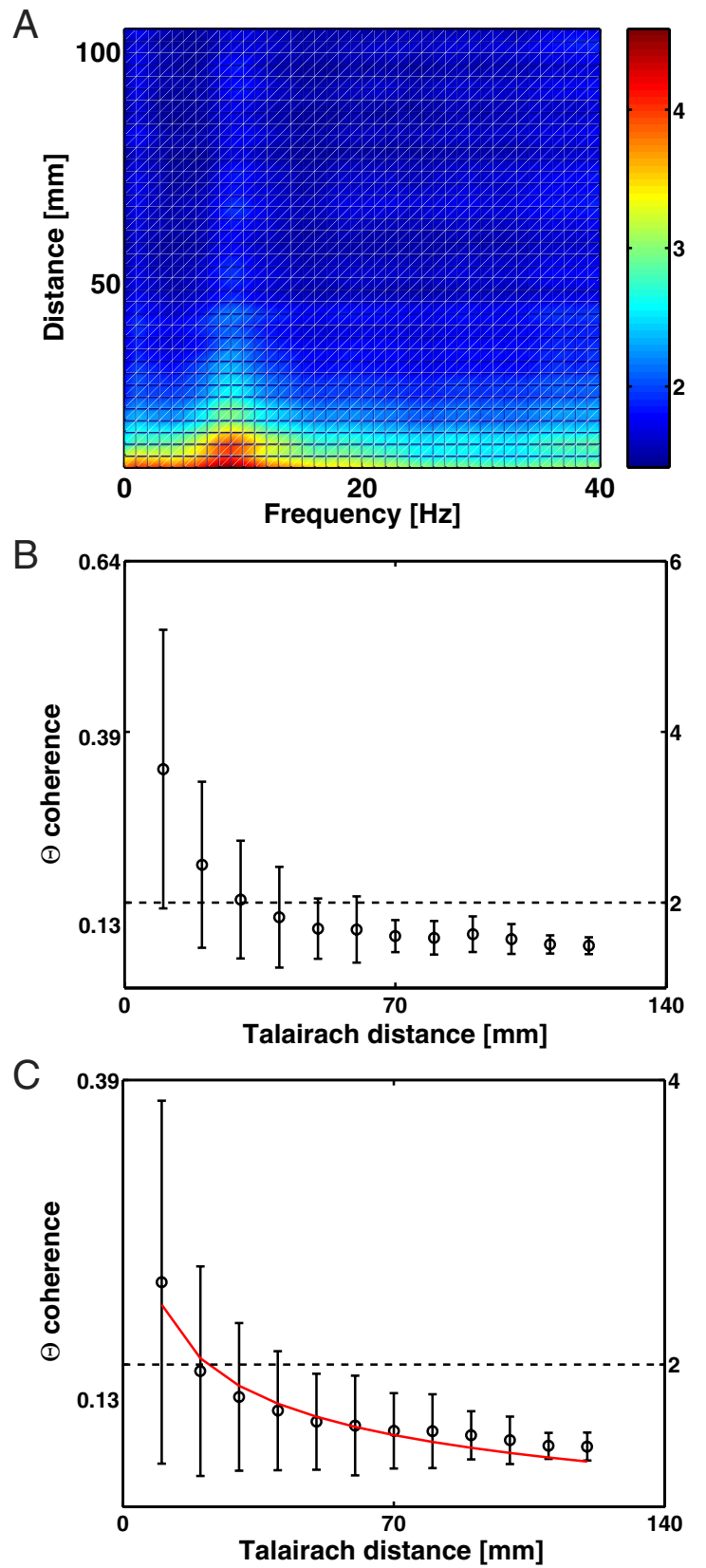

FIG. 3. Theta coherence decreases with interelectrode separation. A: surface plot showing coherence $\left(|\gamma(f)|^{2}\right)$ as a function of distance and frequency averaged across all 1,800 electrode pairs for Participant 1. Theta coherence appears as the most prominent peak. B: theta coherence as a function of Talairach distance for Participant 1. Left axis: actual values. Right axis: $\tanh ^{-1}$ transformed, standardized values. Error bars denote 1 SD (in standardized units). Dashed line indicates the 99th percentile of coherence distribution for 2 independent Gaussian data sets (see METHODS). $C$ : averaged theta coherence for all participants. Red line shows power-law fit, with exponent -0.33 . Axes are as in $B$. Coherence values at large distances are at the median values of coherence for independent Gaussian data sets calculated using df (degrees of freedom $)=24($ see METHODS $)$. 
shallower. We also noted that theta coherence was quite variable at a given distance and that the largest variability of coherence occurred at neighboring electrode locations that were $<20 \mathrm{~mm}$ apart $\left[F_{(1,1640)}=154.1 ; P \ll 0.001\right]$. We conclude for the average cortical site pair (including both gated and nongated sites), theta did not display significant synchronization over distances $>40 \mathrm{~mm}$ and it is highly variable at distances $<20 \mathrm{~mm}$.

We next analyzed the specific subset of sites that are gated by the working-memory task, expecting to see a very different picture. However as seen in Fig. 4, which is a scatterplot of intersite coherence for all gated sites in all participants, the mean coherence for gated sites again tended to decay with distance, whereas the mean levels are higher than the corresponding levels of coherence at a given distance between nongated sites. Indeed, this decay is stronger (distance ${ }^{-0.70 \pm 0.03}$ ) than that for the nongated sites. As can be seen from the scatterplot, the coherence between gated sites also showed large variability. At the smallest interelectrode spacing $(<10 \mathrm{~mm})$ some pairs showed high coherence, significantly different from chance $(P<$ 0.00001; see METHODS), whereas others exhibited low coherence similar to that expected by chance $(P>0.1)$. The variability for pairs of gated sites at separations $<20 \mathrm{~mm}$ was similar to that of nongated sites.

We show an example of pairs of nearby gated sites, recorded using depth electrodes that exhibit spatial differences in spectral coherence (Fig. 5). Figure 5A shows the trial-averaged spectrograms calculated at three depth electrodes in the parahippocampal gyrus from Participant 1, with an interelectrode spacing of $8 \mathrm{~mm}$. Figure $5 B$ shows the location of these sites. All three sites show clear gating. Figure $5 C$ shows the coherence between electrodes 1 and 2 (black and blue symbols) as well as the coherence between electrodes 2 and 3 (blue and red symbols). The former pair shows a very high degree of coherence $(P<0.0001$ compared with Gaussian data, $P<0: 001$ compared with the null distribution from the bootstrap), whereas the coherence of the latter pair is very low and close to that expected by chance $(P>0.5)$.

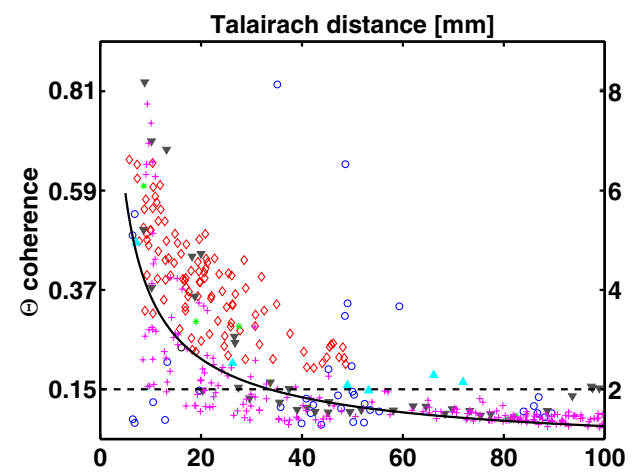

FIG. 4. Gated sites generally do not show long-range coherence. A: scatterplot showing in-task coherence in the theta band $(4-12 \mathrm{~Hz})$ for gated sites in all participants. Left axis: actual values. Right axis: $\tanh ^{-1}$ transformed, standardized values. Black line is a power-law fit with an exponent of -0.71 . Note the large variability at short length scales. Dashed line indicates the 99th percentile of the distribution of coherence of 2 independent Gaussian data sets. Most of the coherence values at larger distances are clustered around the median value of coherence for independent Gaussian data calculated using $\mathrm{df}=24$ (see METHODS).

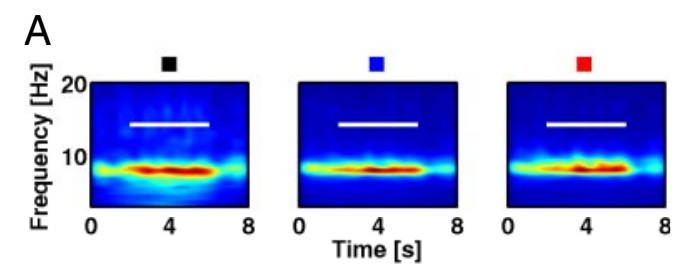

B
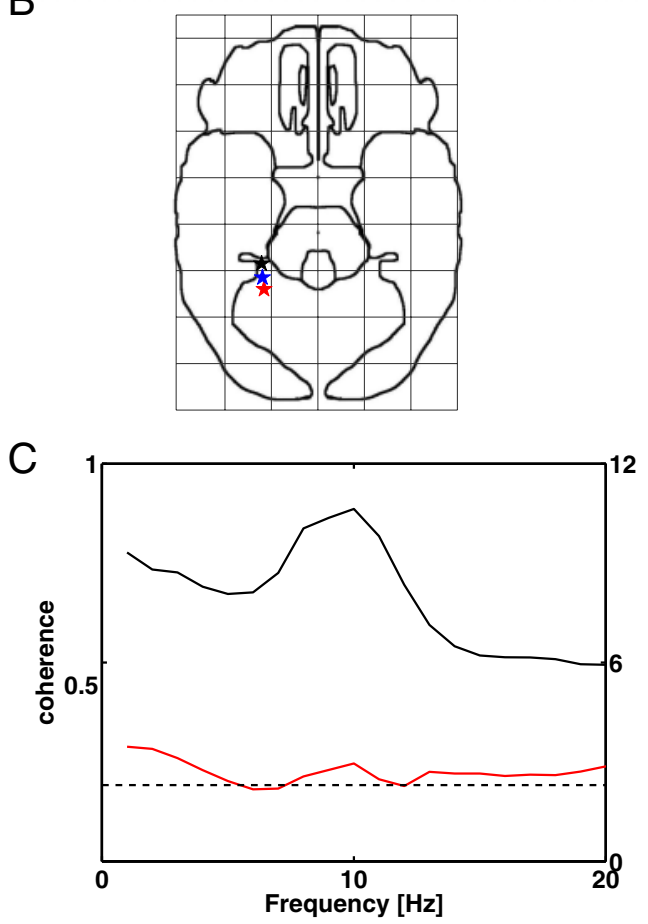

FIG. 5. Theta coherence shows local variability between depth electrode recordings. A: spectrogram of iEEG during the trial (from the onset of first list item until presentation of probe item) shows gated theta activity at all 3 sites in Participant 1. B: inferior view of a standardized brain showing the location of 3 representative depth electrodes with task-modulated theta oscillations [Talairach coordinates: $(\mathrm{L}-\mathrm{R}, \mathrm{A}-\mathrm{P}, \mathrm{I}-\mathrm{S})=(24 ;-43 ;-8),(23 ;-48 ;-4)$, and $(24 ;-37 ;-11)$; blue, red, and black symbols, respectively]. Colored symbols denoting the location of the 3 electrodes correspond to the colored symbol on each spectrogram. $C$ : coherence of the 2 pairs of sites separated by $7 \mathrm{~mm}$ (dashed lines indicates 99\% level of the distribution of coherence of independent Gaussian data). Right axis: corresponding value of coherence in transformed units. Black line is the spectral coherence between the electrode in blue and the electrode in black, whereas the red line corresponds to the spectral coherence between the electrode in blue and the electrode in red. Whereas the first pair exhibits high coherence in the theta band (and high overall coherence) during the entire trial, the second pair shows reduced coherence in the theta band during the same epoch.

This high variability in coherence was observed not only between depth electrodes but also between surface electrodes (Fig. 6). Figure 6A shows the trial-averaged spectrograms calculated at three surface electrodes in the temporal cortex from Participant 10. Again, all three sites show clear gating. Figure $6 B$ shows the location of these three sites. Although sites marked in red and black are about $17 \mathrm{~mm}$ from the site marked in blue, the two pairs show a substantial difference in their coherence in the theta band (Fig. 6C). The above analysis suggests that gated sites, although coordinately regulated by the task, do not generally show significant coherence (Fig. 4). Thus theta oscillations can be local in character. However, examination of the pairwise coherence between gated sites suggests that in some cases, small but significant coherence can 


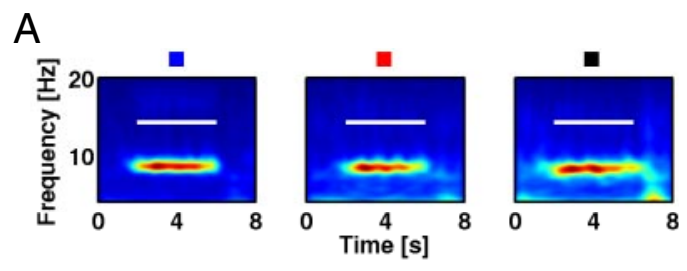

B
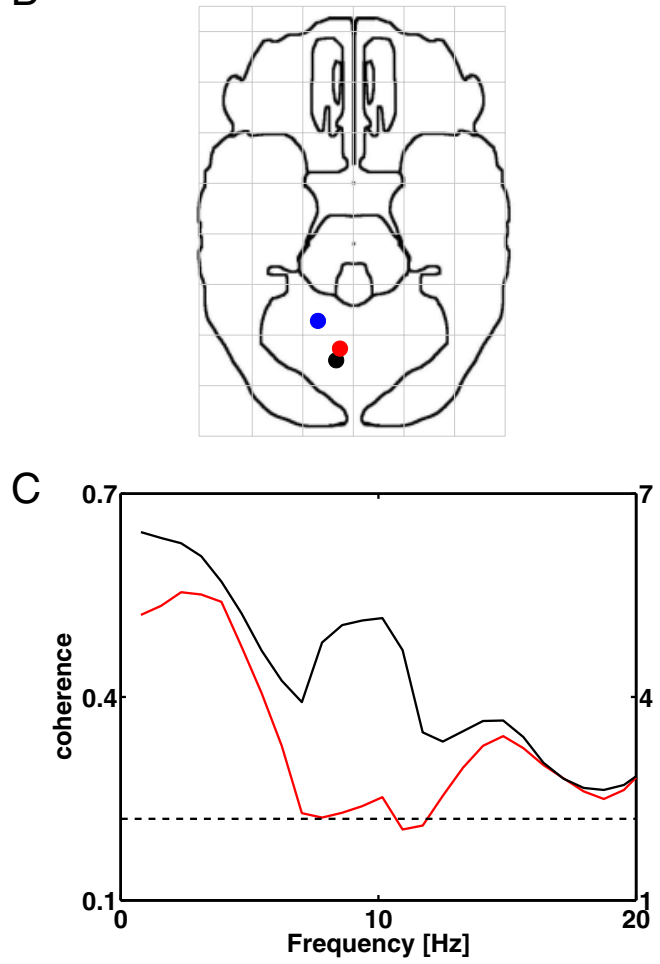

FIG. 6. Theta coherence shows local variability between surface electrodes. A: spectrogram of iEEG during the trial (from the onset of first list item until presentation of probe item) shows gated theta activity at all 3 sites from Participant 10. B: inferior view of a standardized brain showing the location of 3 representative surface electrodes with task-modulated theta oscillations [Talairach coordinates: ( $\mathrm{L}-\mathrm{R}, \mathrm{A}-\mathrm{P}, \mathrm{I}-\mathrm{S})=(4 ;-63 ; 14),(3 ;-79 ; 19)$, and $(5$; $-80 ; 12)$; blue, red, and black symbols, respectively]. Colored symbols denoting the location of the 3 electrodes correspond to the colored symbol on each spectrogram. $C$ : coherence of the 2 pairs of sites separated by about 17 $\mathrm{mm}$ (dashed lines indicates 99\% level of the distribution of coherence of independent Gaussian data). Right axis: corresponding value of coherence in transformed units. Black line is the spectral coherence between the electrode in blue and the one in black, whereas the red line corresponds to the spectral coherence between the electrode in blue and the electrode in red. Although the first pair exhibits high coherence in the theta band during the entire trial, the second pair shows reduced coherence in the theta band during the same epoch. Note that the coherence for the 2 electrode pairs is similar for frequencies $>10$ $\mathrm{Hz}$.

be observed even at distances $>50 \mathrm{~mm}$ (Fig. 4). Although we observed significant coherence of gated sites at large distances in only a single subject, the absolute levels of coherence were quite high, suggesting that the possible synchronization of the iEEG at these sites was robust.

\section{I S C U S S I O N}

Data recorded from 619 sites across ten participants allowed for a detailed characterization of the spatial distribution of theta gating in the human cortex. We demonstrate that gated sites are widespread over the temporal, parietal, and occipital cortex and limbic regions. On the other hand, gated theta appears to be nearly absent in the prefrontal cortex.

The strong presence of theta gating in regions of visual processing was unanticipated based on scalp EEG work (but see Vogel and Machizawa 2004), although this may be explained given the lack of synchronization of sites (see following text) and the limited spatial resolution of scalp EEG. On the other hand, recent work in monkeys using local recordings directly from $\mathrm{V} 4$ also indicates the occurrence of theta during a working-memory task (Lee et al. 2005; Rainer et al. 2004). The involvement of theta in sensory processing is also supported by work in rats. Whisking and sniffing both occur in the theta range and result in corresponding oscillations in the somatosensory and olfactory cortices (Ahrens and Kleinfeld 2004; Fee et al. 1997; Kleinfeld et al. 2002). Taken together the results across species make a strong case that theta oscillations in the sensory cortex play an important role in working memory. The fact that theta oscillations in occipital cortex are gated by contingencies of the task make the further point that early sensory processing is not driven exclusively by the visual input, but also depends on preestablished task requirements, such as attention (Fries et al. 2001) or, in this case, working memory. It will also be important to establish whether these findings are particular to the visual working-memory paradigm that we have used, or are more generally involved in memory tasks with multiple modalities, using either intracranial or scalp EEG recordings.

Although we sampled a total of 182 sites in the frontal cortex, we found only two clear examples (in two subjects total) of gated theta in this region. This may seem surprising in view of the extensive evidence for the role of prefrontal cortex in working memory. Consistent with our lack of finding of prefrontal theta in the Sternberg task, Rizzuto et al. (2003) observed that stimulus-induced reset of theta phase was observed in many brain regions, but not in the prefrontal cortex. In considering the possible interpretations of these findings, we note that theta oscillations may make possible the coding of multiple items into short-term memory by a phase-coding scheme (see below). It is thus instructive to consider specifically the regions implicated in tasks where the fundamental capacity limit of short-term memory can be explicitly examined. Neuropsychological work implicates the parietal lobe as the principal brain region where lesions affect the capacity limit of short-term memory (D'Esposito and Postle 1999; Postle et al. 2004; Shallice and Vallar 1990). Consistent with these lesion data, scalp EEG components recorded over parietal cortex have recently been shown to vary with memory load (Vogel and Machizawa 2004). Thus one possible interpretation of our results is that a posterior network, which includes the parietal lobe, uses a theta-organized system to maintain information over short delays, and that the role of the prefrontal cortex is to provide executive control of the task, for instance in setting up stimulus response contingencies (Miller and Cohen 2001).

Although widespread throughout the cortex, gated sites did not generally exhibit significant coherence even though they were jointly engaged by the task (Fig. 4). This finding suggests that local networks coordinately engaged by the workingmemory tasks can independently generate theta activity. As a general measure of how synchronization depends on interelectrode separation, we plotted coherence as a function of the 
distance between electrodes (Figs. 3 and 4). When we considered all sites, irrespective of whether they were gated, we found that coherence decreases as a power-law function of distance (Bullock et al. 1995; Menon et al. 1996). Our expectation was that the degree of long-range coherence would be much higher when we considered only gated sites because these sites are clearly jointly regulated by the task. We found, however, that the falloff of coherence with distance was similar to that for all sites, and that distant gated sites were generally incoherent. On the other hand, closely spaced gated sites are often, but not necessarily, coherent. The absolute level of coherence at nearby sites was substantially higher during the task. We emphasize that, although our results strongly point to the fact that distant sites are generally not synchronized, the results do not rule out the possibility of long-range synchronization in rare cases. In Fig. 4, there are a few distant locations (separated by as much as $50 \mathrm{~mm}$ ) that are synchronized. Because these happen to be all from the same participant, we cannot speak to the generality of this finding. Additionally, it may be possible that some of the synchronized pairs of sites that appear to be close in Talairach space may actually be quite distant in cortical space, i.e., when the cortex is unfolded. Because of technical limitations, we did not have high-resolution MR images that could be used to construct an inflated map of the cortex and obtain true cortical distances between different electrodes.

The general lack of coherence implies that cortex is quite different from the hippocampus in terms of theta synchronization. The rodent hippocampus receives a rhythmic input from the medial septal nucleus that oscillates at theta (Buzsaki 2002). In addition, intrahippocampal circuitry also appears to generate theta-frequency oscillations (Buzsaki 2002; Kocsis et al. 1999). The large-scale synchronization of theta oscillations in the hippocampus seems to arise from the interplay of these extrinsic and intrinsic oscillators. In contrast, it appears that cortical subregions generally oscillate independently.

The ability of cortical sites to locally generate theta is supported by work on cortical slices demonstrating the capability of local theta generation. Connors and colleagues made cortical slices in which layer 5 was isolated and showed that facilitating $N$-methyl-D-aspartate transmission by lowering $\mathrm{Mg}^{2+}$ concentration resulted in theta-frequency oscillations in the field potential (Flint and Connors 1996; Silva et al. 1991). A recent study showed the existence of a population of interneurons that generate synchronous theta-frequency activity on cholinergic activation and pace pyramidal cell output (Blatow et al. 2003). Imaging studies of neocortical slices using voltage-sensitive dyes during carbachol application show that the local field potential (LFP) oscillates in the theta-frequency range (Bao and $\mathrm{Wu} 2003$ ). Interestingly, these oscillations are synchronous across only a $300-\mu \mathrm{m}$ range, suggesting that the generation mechanism is local. Based on these results, the idea that the theta oscillations we have observed in human cortex are locally generated seems quite plausible. Such local oscillations would explain the observed lack of long-range synchronization.

The rapid decay of coherence with interelectrode distance (Bullock et al. 1995; Menon et al. 1996) and high variability (Shen et al. 1999) is typical of intracranial recordings from humans. However, in all of these studies, the coherence was not calculated while the participant performed a cognitive task, which might be expected to enhance the coherence between different regions that participate in the task. Moreover, these earlier studies did not report a spectral peak (signifying a large-amplitude oscillation) in any frequency band. In our study, the iEEG is characterized by a large spectral peak in the theta-frequency range, which is tightly linked to the task. Thus one might expect a priori that such task-related oscillations might be synchronized across cortical regions. However, we found that the overall pattern of coherence was similar to that observed in the earlier studies. On the other hand, there have been previous indications of long-range theta synchronization in scalp EEG during memory tasks (Mima et al. 2001; Sarnthein et al. 1998). In particular, Sarnthein et al. (1998) found increased coherence in the theta-frequency range between frontal and parietal electrodes during a memory period over a control period during a working-memory task. However, the absolute levels of coherence were rather low and the authors did not test whether spectrally matched random Gaussian noise would lead to similar levels of coherence. Moreover, some of this coherence could be explained by the fact that scalp EEG recordings sample larger areas of the cortical surface arising from volume conduction and blurring attributed to the skull (Nunez et al. 1997; Srinivasan et al. 1998). Thus sensors several centimeters apart on the scalp can become partially synchronized, even though the underlying cortical sources are not. However, although our work indicates that distant sites are generally not synchronized in the theta-frequency range, such synchronization may well occur in rare cases and contribute to the changes in long-range synchronization changes observed in scalp EEG recordings. Moreover, our study had only two subjects with simultaneous electrode placement in both frontal and parietal cortex and synchronization between special sites in these cortical areas could have been missed as a result of insufficient spatial sampling.

\section{Possible function of theta}

The clearest evidence regarding the function of theta comes from recordings of hippocampal place cells in rat. As a rat runs through the place field, the average phase of spikes on each progressive theta cycle shifts forward (O'Keefe and Recce 1993; Skaggs et al. 1996). The information content of this phase coding is demonstrated by the ability to reconstruct position from ensembles of simultaneously recorded cells; the accuracy of reconstruction is much better when phase is taken into consideration than when only rate information is used (Jensen and Lisman 1998). One interpretation of the phase precession is that it represents cued recall of the coming positions along the track, based on sequence information stored in hippocampal synapses (Jensen and Lisman 1998; Lisman 1999; Tsodyks et al. 1996). From the perspective of the present discussion, the key idea here is that theta provides an absolute phase reference; thus the earlier the phase of a neuron's spiking, the closer the position represented by that cell. This concept of theta phase coding could also be important for the cortical theta that occurs during the Sternberg task. The cells representing different items stored in working memory during the Sternberg task could be active at different phases (Jensen and Lisman 2005; Lisman and Idiart 1995). Moreover, the existence of an absolute phase reference could provide the basis for recall of list items in order (Lisman 2005). Recent 
evidence for theta-frequency oscillatory in monkey V4 during a working-memory task (Lee et al. 2005; Rainer et al. 2004) suggests that these speculative ideas may soon be experimentally testable. An alternative interpretation of our finding of gated theta combined with local coherence of these gated sites would be that gated theta reflects a disengagement with the ongoing task. However, this interpretation is unlikely given the study of Sederberg et al. (2003), which showed that theta oscillations predicted subsequent recall of words in a list, indicating the importance of theta for memory.

Our results rule out the hypothesis that theta-gated sites are synchronized throughout the working-memory period. However, a model not ruled out by our data is that such sites are transiently synchronized for a short period relative to the task duration (about $1 \mathrm{~s}$ ). Such brief synchronization would not be detected by our measure of coherence, which averages over the entire task duration. Indeed several lines of evidence point to the existence of short periods of theta (Caplan et al. 2001; Kahana et al. 1999) and of changes in theta coherence (Macrides et al. 1982). Additionally, the faster decay of coherence as a function of spatial separation among gated sites in our data set might arise from brief periods of high synchronization in an otherwise weakly correlated background. Thus theoretical and experimental work will be needed to explore the idea that distant cortical regions function independently except during brief periods of information transfer.

\section{G R A N T S}

This work was supported by National Institutes of Health Grants MH-61975 and MH-55687 to M. J. Kahana and NS-27337 to J. E. Lisman, a National Alliance for Research on Schizophrenia and Depression Young Investigator award to S. Raghavachari, and the W. M. Keck Foundation.

\section{REFERENCES}

Ahrens KF and Kleinfeld D. Current flow in vibrissa motor cortex can phase-lock with exploratory rhythmic whisking in rat. J Neurophysiol 92: 1700-1707, 2004.

Bao W and Wu J-Y. Propagating wave and irregular dynamics: spatiotemporal patterns of cholinergic theta oscillations in neocortex in vitro. $\mathrm{J} \mathrm{Neu}$ rophysiol 90: 333-341, 2003.

Blatow M, Rozov A, Katona I, Hormuzdi SG, Meyer AH, Whittington MA, Caputi A, and Monyer H. A novel network of multipolar bursting interneurons generates theta frequency oscillations in neocortex. Neuron 38 : 805-817, 2003.

Bullock TH, Buzsaki G, and McClune MC. Coherence of compound field potentials reveals discontinuities in the CA1-subiculum of the hippocampus in freely moving rats. Neuroscience 38: 609-619, 1990.

Bullock TH, McClune MC, Achimowicz JZ, Iragui-Madoz VJ, Duckrow RB, and Spencer SS. EEG coherence has structure in the millimeter domain: subdural and hippocampal recordings from epileptic patients. Electroencephalogr Clin Neurophysiol 95: 161-177, 1995.

Buzsaki G. Theta oscillations in the hippocampus. Neuron 33: 325-340, 2002.

Caplan JB, Madsen JR, Raghavachari S, and Kahana MJ. Distinct patterns of brain oscillations underlie two basic parameters of human maze learning. J Neurophysiol 86: 366-380, 2001.

Caplan JB, Madsen JR, Schulze-Bonhage A, Aschenbrenner-Scheibe R, Newman EL, and Kahana MJ. Human theta oscillations related to sensorimotor integration and spatial learning. J Neurosci 23: 4726-4736, 2003.

D'Esposito M and Postle BR. The dependence of span and delayed-response performance on prefrontal cortex. Neuropsychologia 37: 1303-1315, 1999.

Fee MS, Mitra PP, and Kleinfeld D. Central versus peripheral determinants of patterned spike activity in rat vibrissa cortex during whisking. J Neurophysiol 78: 1144-1149, 1997.

Flint AC and Connors BW. Two types of network oscillations in neocortex mediated by distinct glutamate receptor subtypes and neuronal populations. J Neurophysiol 75: 951-957, 1996.
Fries P, Reynolds JH, Rorie AE, and Desimone R. Modulation of oscillatory neuronal synchronization by selective visual attention. Science 291: 1560$1563,2001$.

Hölscher C, Anwyl R, and Rowan MJ. Stimulation on the positive phase of hippocampal theta rhythm induces long-term potentiation that can be depotentiated by stimulation on the negative phase in area CA1 in vivo. J Neurosci 17: 6470-6477, 1997.

Howard MW, Rizzuto DS, Caplan JB, Madsen JR, Lisman J, Aschenbrenner-Scheibe R, Schultze-Bonhage A, and Kahana MJ. Gamma oscillations increase with memory load in humans. Cereb Cortex 13: 1369-1374, 2003.

Huerta PT and Lisman J. Bidirectional synaptic plasticity induced by a single burst during cholinergic theta oscillation in CA1 in vitro. Neuron 15: 1053-1063, 1995.

Jensen $\mathbf{O}$ and Lisman JE. An oscillatory short-term memory buffer model can account for data on the Sternberg task. J Neurosci 18: 10688-10699, 1998.

Jensen $\mathbf{O}$ and Lisman JE. Hippocampal sequence-encoding driven by a cortical multi-item working memory buffer. Trends Neurosci 28: 67-72, 2005.

Kahana MJ. The cognitive correlates of human brain oscillations. J Neurosci In press.

Kahana MJ, Sekuler R, Caplan JB, Kirschen M, and Madsen JR. Human theta oscillations exhibit task dependence during virtual maze navigation. Nature 399: 781-784, 1999.

Kleinfeld D, Sachdev RNS, Merchant LM, Jarvis MR, and Ebner FF. Adaptive filtering of vibrissa input in motor cortex of rat. Neuron 34: 1021-1034, 2002.

Kocsis B, Bragin A, and Buzsaki G. Interdependence of multiple theta generators in the hippocampus: a partial coherence analysis. J Neurosci 19: 6200-6212, 1999.

Lee H, Simpson G, Logothetis N, and Rainer G. Phase locking of single neuron activity to theta oscillations during working memory in monkey extrastriate visual cortex. Neuron 45: 147-156, 2005.

Leung LW and Borst JG. Electrical activity of the cingulate cortex. I. Generating mechanisms and relations to behavior. Brain Res 407: 68-80, 1987.

Lisman J. Relating hippocampal circuitry to function: recall of memory sequences by reciprocal dentate-CA3 interactions. Neuron 22: 233-242, 1999.

Lisman J. The theta/gamma discrete phase code occurring during the hippocampal phase precession may be a more general brain coding scheme. Hippocampus 15: 913-922, 2005.

Lisman JE and Idiart MA. Storage of $7 \pm 2$ short-term memories in oscillatory subcycles. Science 267: 1512-1515, 1995.

Macrides F, Eichenbaum HB, and Forbes WB. Temporal relationship between sniffing and the limbic theta rhythm during odor discrimination reversal learning. J Neurosci 2: 1705-1717, 1982.

Menon V, Freeman WJ, Cutillo BA, Desmond JE, Ward MF, Bressler SL, Laxer KD, Barbaro N, and Gevins AS. Spatio-temporal correlations in human gamma band electrocorticograms. Electroencephalogr Clin Neurophysiol 98: 89-102, 1996.

Miller EK and Cohen JD. An integrative theory of prefrontal cortex function. Annu Rev Neurosci 24: 167-202, 2001.

Mima T, Oluwatimilehin T, Hiraoka T, and Hallett M. Transient interhemispheric neuronal synchrony correlates with object recognition. $\mathrm{J} \mathrm{Neu}$ rosci 21: 3942-3948, 2001.

Mitra PP and Pesaran B. Analysis of dynamic brain imaging data. Biophys J 76: 691-708, 1999.

Nunez PL, Srinivasan R, Westdorp AF, Wijesinghe RS, Tucker DM, Silberstein RB, and Cadusch PJ. EEG coherency I: statistics, reference electrode, volume conduction, Laplacians, cortical imaging, and interpretation at multiple scales. Electroencephalogr Clin Neurophysiol 100: 499$515,1997$.

O'Keefe J and Recce ML. Phase relationship between hippocampal place units and the EEG theta rhythm. Hippocampus 3: 317-330, 1993.

Pavlides C, Greenstein YJ, Grudman M, and Winson J. Long-term potentiation in the dentate gyrus is induced preferentially on the positive phase of theta-rhythm. Brain Res 439: 383-387, 1988.

Postle BR, Awh E, Jonides J, Smith EE, and D'Esposito M. The where and how of attention-based rehearsal in spatial working memory. Brain Res Cogn Brain Res 20: 194-205, 2004. 
Raghavachari S, Kahana MJ, Rizzuto D, Caplan J, Kirschen M, Bourgeois B, Madsen J, and Lisman J. Gating of human theta oscillations by a working memory task. J Neurosci 21: 3175-3183, 2001.

Rainer G, Lee H, Simpson G, and Logothetis NK. Working-memory related theta frequency range oscillations observed in monkey extrastriate visual cortex. Neurocomputing 58-60: 965-969, 2004.

Rizzuto DS, Madsen JR, Bromfield E, Schulze-Bonhage A, Seelig D, Aschenbrenner-Scheibe R, and Kahana MJ. Reset of human neocortical oscillations by a working memory task. Proc Natl Acad Sci USA 100: 7931-7936, 2003.

Sarnthein J, Petsche H, Rappelsberger P, Shaw GL, and von Stein A. Synchronization between prefrontal and posterior association cortex during human working memory. Proc Natl Acad Sci USA 95: 7092-7096, 1998.

Sederberg P, Kahana M, Howard M, Donner E, and Madsen J. Theta and gamma oscillations during encoding predict subsequent recall. J Neurosci 23: 10809-10814, 2003.

Shallice $\mathbf{T}$ and Vallar G. The impairment of auditory verbal short-term storage. In: Neuropsychological Impairments of Short-Term Memory, edited by Vallar G and Shallice T. New York: Cambridge Univ. Press, 1990, p. $11-53$.

Shen B, Nadkarni M, and Zappulla RA. Spectral modulation of cortical connections measured by EEG coherence in humans. Clin Neurophysiol 110: $115-125,1999$.
Silva LR, Amitai Y, and Connors BW. Intrinsic oscillations of neocortex generated by layer 5 pyramidal neurons. Science 251: 432-435, 1991.

Skaggs WE, McNaughton BL, Wilson MA, and Barnes CA. Theta phase precession in hippocampal neuronal populations and the compression of temporal sequences. Hippocampus 6: 149-172, 1996.

Srinivasan R, Nunez PL, and Silberstein RB. Spatial filtering and neocortical dynamics: estimates of EEG coherence. IEEE Trans Biomed Eng 45: 814-826, 1998.

Sternberg S. High-speed scanning in human memory. Science 153: 652-654, 1966.

Thomson D and Chave A Jackknifed error estimates for spectra, coherences and transfer functions. In: Advances in Spectrum Analysis and Array Processing. New York: Prentice Hall, 1991, vol. 1, p. 58-113.

Tsodyks MV, Skaggs WE, Sejnowski TJ, and McNaughton BL. Populations dynamics and theta rhythm phase precession of hippocampal place cell firing: a spiking neuron model. Hippocampus 6: 271-280, 1996.

Vogel EK and Machizawa MG. Neural activity predicts individual differences in visual working memory capacity. Nature 428: 748-751, 2004.

Winson J. Loss of hippocampal theta rhythm results in spatial memory deficit in the rat. Science 201: 160-163, 1978.

Zaveri HP, Williams WJ, Sackellares JC, Beydoun A, Duckrow RB, and Spencer SS. Measuring the coherence of intracranial electroencephalograms. Clin Neurophysiol 110: 1717-1725, 1999. 